\title{
3efferson.
}

JHN Journal

Volume 11 | Issue 1

Article 1

Winter 2016

\section{A Case of Acute Disseminated Encephalomyelitis in a MIddle-Aged Adult}

Nicole Mahdi, MD

Department of Neurological Surgery, Thomas Jefferson University, nicole.mahdi@jefferson.edu

Peter Abdelmalik, MD

Department of Neurological Surgery, Thomas Jefferson University, peter.abdelmalik@jefferson.edu

Mark T. Curtis, MD

Department of Pathology, Thomas Jefferson University, mark.curtis@jefferson.edu

Barak Bar, MD

Department of Neurological Surgery, Thomas Jefferson University, barak.bar@jefferson.edu

Follow this and additional works at: https://jdc.jefferson.edu/jhnj

Let us know how access to this document benefits you

\section{Recommended Citation}

Mahdi, MD, Nicole; Abdelmalik, MD, Peter; Curtis, MD, Mark T.; and Bar, MD, Barak (2016) "A Case of Acute Disseminated Encephalomyelitis in a MIddle-Aged Adult," JHN Journal: Vol. 11 : Iss. 1 , Article 1.

DOI: https://doi.org/10.29046/JHNJ.011.1.001

Available at: https://jdc.jefferson.edu/jhnj/vol11/iss1/1

This Article is brought to you for free and open access by the Jefferson Digital Commons. The Jefferson Digital Commons is a service of Thomas Jefferson University's Center for Teaching and Learning (CTL). The Commons is a showcase for Jefferson books and journals, peer-reviewed scholarly publications, unique historical collections from the University archives, and teaching tools. The Jefferson Digital Commons allows researchers and interested readers anywhere in the world to learn about and keep up to date with Jefferson scholarship. This article has been accepted for inclusion in JHN Journal by an authorized administrator of the Jefferson Digital Commons. For more information, please contact: JeffersonDigitalCommons@jefferson.edu. 


\section{A Case of Acute Disseminated Encephalomyelitis in a Middle-Aged Adult}

\author{
Nicole Mahdi, MD¹; Peter Abdelmalik, MD; Mark T. Curtis, MD²; Barak Bar, MD \\ ${ }^{1}$ Department of Neurological Surgery, Thomas Jefferson University, Philadelphia, PA \\ 2Department of Pathology, Thomas Jefferson University, Philadelphia, PA
}

\section{BACKGROUND}

Acute disseminated encephalomyelitis (ADEM) is a monophasic inflammatory demyelinating disorder of the white matter that is often preceded by viral infection or recent vaccination. Encephalopathy and focal neurological deficits usually manifest one to three weeks after a prodromal illness with neurologic decline progressing rapidly over days to weeks. Approximately $25 \%$ of patients will develop multiple sclerosis (MS) within five years of initial presentation of ADEM but the majority of individuals do not progress beyond three months. ${ }^{4}$ ADEM is most commonly seen in children and young adults, where prognosis is favorable, but very few cases have been reported of middle-aged or elderly patients. The clinical course of these patients as compared to younger patients with ADEM is unclear. Here we present a case of ADEM in a middle-aged adult that recovered well after treatment with high-dose corticosteroids.

\section{CASE SUMMARY}

A 62-year-old man with a history of hypertension initially presented with progressive development of gait dysfunction, urinary incontinence, and encephalopathy over the course of two weeks following four days of a gastrointestinal illness. Upon presentation to an outside hospital, he was non-verbal and due to his depressed mental status required intubation. He underwent an MRI of the brain on the first hospital day (HD) that revealed extensive supratentorial white matter hyperintensities extending from the periventricular region through subcortical fibers without contrast enhancement (see figure 1). Thus he was started on 250 milligrams (mg) of intravenous (IV) methylprednisolone every 6 hours for presumed ADEM. He also underwent a lumbar puncture on HD \#1 prior to administration of IV steroids that revealed an opening pressure of 20, with cerebrospinal fluid (CSF) containing 0 red blood cells, 47 white blood cells of which $85 \%$ were lymphocytes, glucose of 52 and protein of 114 He was empirically treated with acyclovir and ceftriaxone for a total 5 days, which were discontinued afterCSF bacterial cultureswerenegative. Blood cultureswerealso negativeand a transthoracic echocardiogram was negative for evidence of vegetation to suggest endocarditis. Various viral titers including herpes simplex virus (HSV), Epstein Bar virus (EBV), varicella zoster virus (VZV), and cytomegalovirus (CMV) were negative, as were serologies for CSF Lyme and Cryptococcus neoformans. In addition, CSF oligoclonal bands and myelin basic protein were negative. Serum inflammatory markers including ANA, $C$ reactive protein, and sedimentation rate were not elevated. EEG showed diffuse slowing suggestive of moderate diffuse cerebral dysfunction without evidence of seizures or epileptiform activity.

Despite treatment with high dose steroids for approximately 6 days at the outside hospital, his mental status did not improve and thus he was transferred to our facility to the neurological intensive care unit for further management. Upon arrival, the patient's GCS was 10T, with spontaneous eye opening, ability to localize to pain in the left upper extremity, and intact brain stem reflexes. Additionally, he displayed triple flexion in his bilateral lower extremities and decorticate posturing in his right upper extremity. A repeat lumbar puncture performed on HD \#8 demonstrated a normal opening pressure, with CSF containing 0 red blood cells, 9 white blood cells of which $94 \%$ were lymphocytes, glucose of 79 and protein of 100. CSF bacterial cultures were negative, as well as no evidence of active HSV or EBV infection. CSF cytology revealed the presence of white blood cells but was negative for malignancy. RPR was also negative. Two subsequent MRIs of the brain were performed on HD \#13 and HD \#21 showing no significant change in the appearance of the prior white matter lesions and no new contrast enhancing lesions. In addition, an MRI of the cervical and thoracic spine with contrast performed on HD \#22 did not show any prior or new enhancing white matter lesions. With continued treatment with high dose IV steroids at $250 \mathrm{mg}$ of IV methylprednisolone every 6 hours for another 7 days, his mental status improved and thus he was safely extubated on HD \#10 and continued on a taper of oral prednisone. He underwent a brain biopsy on HD \#13 that revealed multiple small foci of macrophage accumulation and widespread white matter inflammation secondary to demyelination (figure 2). He was subsequently discharged on HD \#63 to acute rehab on a prolonged oral steroid taper. His physical exam on discharge was notable for a GCS of 15 , fully oriented, full strength on the left, and a residual right hemiparesis. Upon outpatient follow up four months later, he made further improvements, now ambulating without assistance, cognitively back to his baseline, and independent in his activities of daily living, with a modified Rankin score (mRS) of 0 . He underwent a follow up MRI brain five months later that showed overall improvement in the diffuse white matter changes previously seen on initial presentation (see figure 1). 

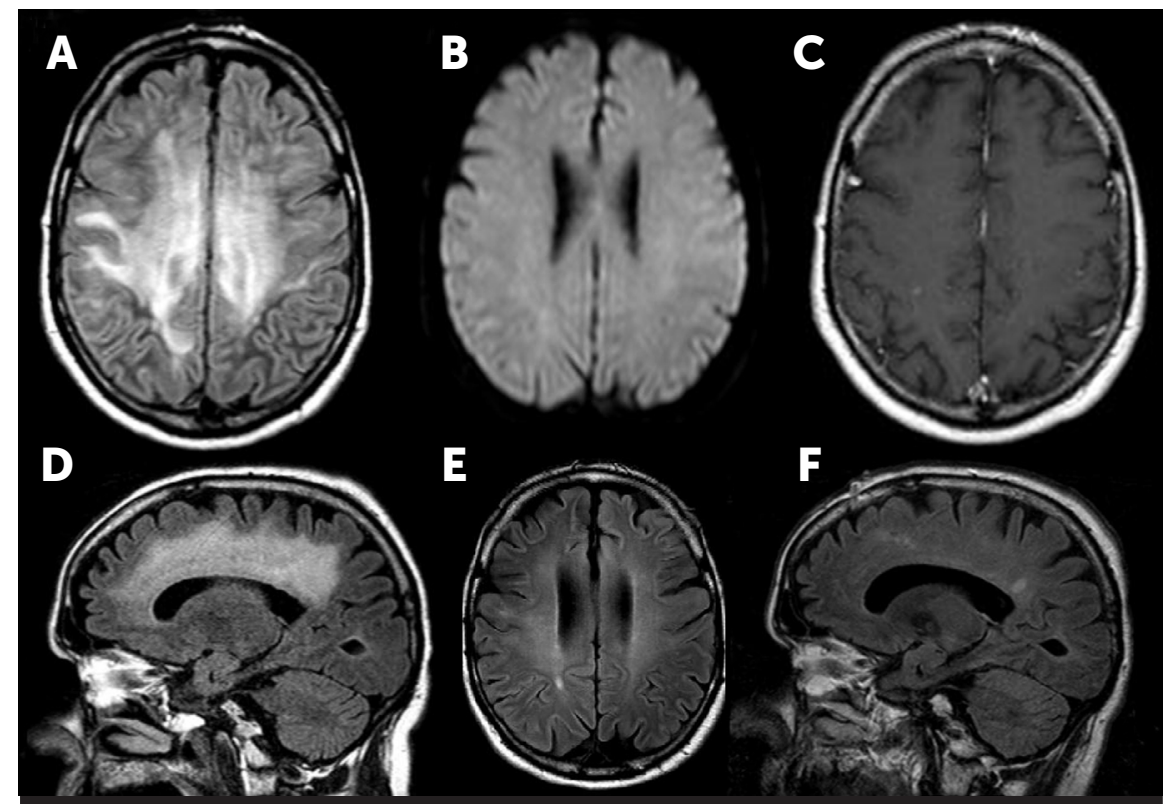

$\mathbf{E}$
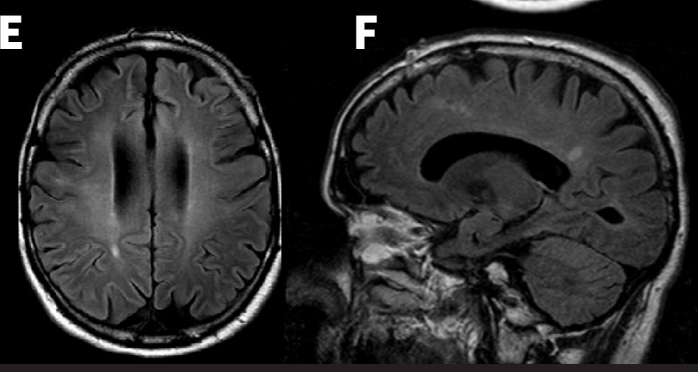

Figure 1

MRI Imaging on admission A - D in comparison to those obtained on 7

month follow up E \& F. A. Axial t2/FLAIR showing supratentorial WM hyperintensities

extending from the periventricular region through the subcortical fibers. B. Axial

view diffusion- weighted imaging showing no restricted diffusion to suggest acute

infarction. C. Axial T1 post-contrast image showing no contrast enhancement

of the T2/FLAIR lesions. D. Sagittal T2/FLAIR image showing diffuse periventricular

WM intensities. E. Axial T2/ FLAIR showing interval improvement $\&$ resolution

of the periventricular WM lesions. F. Sagittal T2/FLAIR also demonstrating the

improvement of the periventricular WM lesions.

\section{DISCUSSION}

ADEM is theorized to be an immunologically mediated demyelinating disease triggered by a febrile illness or recent vaccination, eliciting an inflammatory response affecting the central nervous system (CNS). Possible mechanisms may include either molecular mimicry or direct inflammatory damage to myelinated neurons. ${ }^{2}$ The prevalence of ADEM is higher in children and young adults and thought to be related to the increased frequency of viral infections and vaccination in this patient population. ${ }^{6}$ The demonstration of multifocal hyperintensities most often affecting the subcortical white matter on fluid attenuated inversion recovery (T2/FLAIR) MRI of the brain make the identification of ADEM difficult given various other inflammatory, infectious, and rheumatologic disorders that have similar clinical and radiological presentations. Given such ambiguity, the International Pediatric Multiple Sclerosis Study Group proposed a consensus definition for the pediatric population with the mandatory inclusion of encephalopathy in the criteria for diagnosis. ${ }^{2}$ Despite this useful aid in identifying young patients, there still remains no clear diagnostic criterion for ADEM in adults, and therefore older individuals are more difficult to prognosticate given the paucity of reported cases and outcomes following standard ADEM therapies such as high dose corticosteroids, intravenous immunoglobulin (IVIG), and plasmapheresis (PLEX). Our patient presented with a suggestive clinical history of symptoms, physical exam and radiological signs, along with a CSF profile and histopathology suggestive of ADEM. Given both the therapeutic and prognostic implications of considering this a case as the first demyelinating event of MS, it important to make clear that this most likely does not represent MS given the initial subacute presentation of encephalopathy, the presence of multifocal lesions with illdefined margins, and pathology showing widespread inflammation. In contrast, MS often presents with distinct episodes of focal neurological deficits with concordant confluent demyelinating lesions of varying ages appearing ovoid in shape, often affecting the white matter more than gray matter, the latter of which is seen more commonly in $\mathrm{ADEM}^{4}$. CSF studies reveal the persistent presence of oligoclonal bands more frequently in $M S$ in comparison to $A_{D E M}{ }^{5}$. Furthermore in this patient, subsequent MRIs confirmed no evidence of new contrast enhancing lesions, with follow up MRI five months later showing resolution of a monophasic demyelinating event with concomitant clinical resolution of neurological deficits. Additionally, due to the negative aforementioned serologies and inflammatory markers, as well as the lack of systemic symptoms, this case likely does not represent a manifestation of an underlying infectious, neoplastic, or rheumatologic condition that may mimic a demyelinating CNS insult.

A small case series of middle-aged adults presenting with ADEM has been described by Wang et al ${ }^{1}$, in which all three patients had a single episode of focal neurological deficits and encephalopathy with typical MRI findings, and clinically improved with steroid treatment. Although typically reserved for more fulminant forms of ADEM, there have also been individual reports of the concomitant use of steroids along with IVIG and PLEX in the treatment of ADEM in older patients with favorable outcomes, suggesting that this presentation in older adults may in fact be of the similar pathophysiology that is well-described in children and young adults. ${ }^{3,7}$

ADEM rarely presents in the middle-aged to elderly adult and due to the paucity of cases reported in the literature, the prognosis in this age group is unknown. We present a case of ADEM in a middleaged adult in which the patient had an excellent response to treatment with high dose steroids, resulting in a remarkable neurological recovery. Thus it behooves us to treat suspected cases of ADEM in an adult patient aggressively, as outcome may be favorable. 


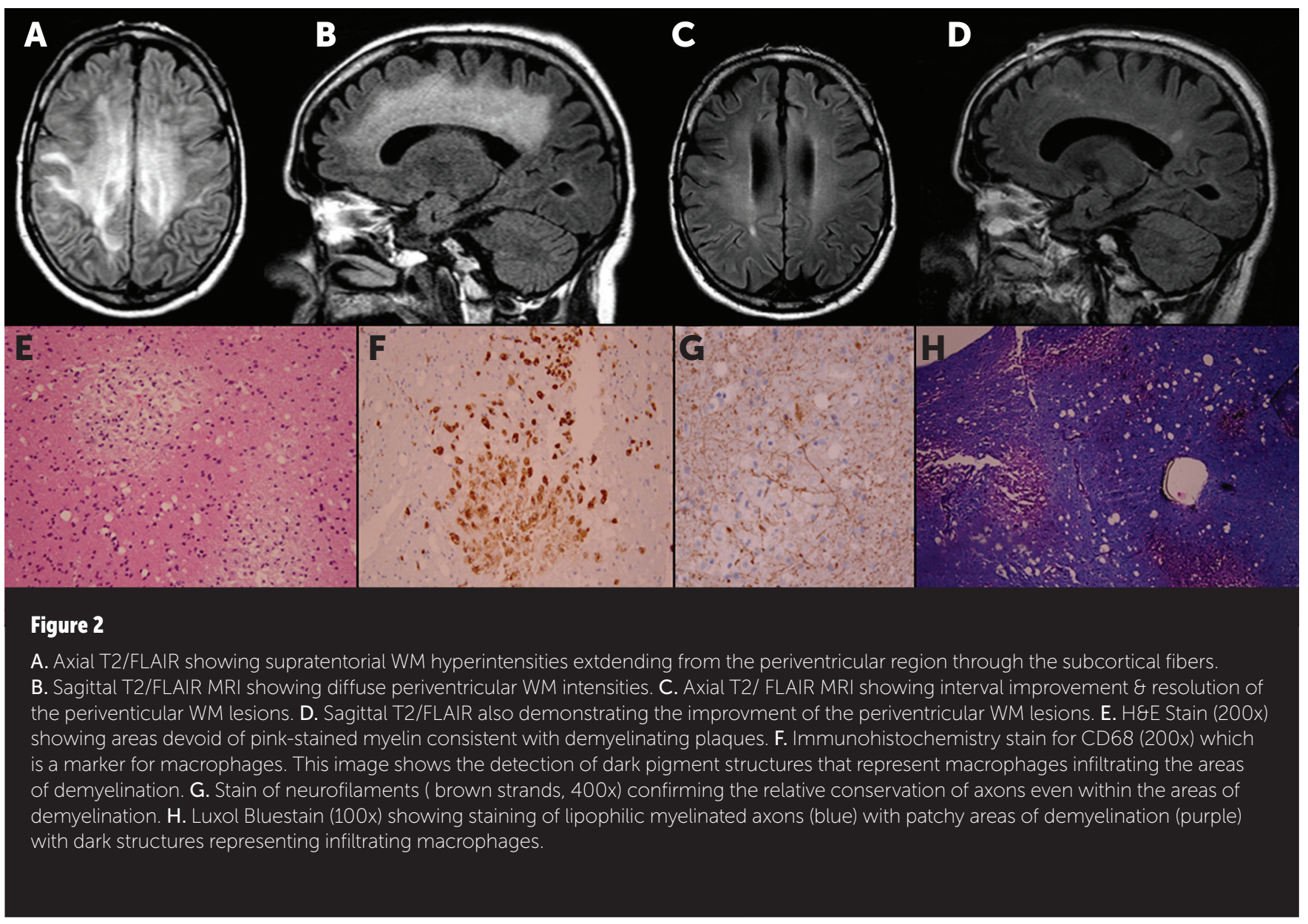

\section{REFERENCES}

1. Wang PN, Fuh JL, Liu HC, Wang SJ. Acute disseminated encephalomyelitis in middle-aged or elderly patients. Eur Neurol 1996;36:219-223.

2. Marin SE, Callen D. The magnetic resonance imaging appearance of monophasic acute disseminated encephalomyelitis: an update post application of the 2007 consensus criteria. Neuroimag Clin N Am 2013; 23:245-266.

3. Otten CE, Creutzfeldt CJ. Fulminant acute disseminated encephalomyelitis presenting in an adult. JAMA Neurology 2014; 71(5):648-649.
4. Rahmlow MR, Kantarci, O. Fulminant demyelinating diseases. The Neurohospitalist 2013; 3(2):81-91.

5. de Seze J, et al. Acute fulminant demyelinating disease. Arch Neurol 2007; 64(10); 1426-32.

6. Alexander M, Murthy JMK. Acute disseminated encephalomyelitis: treatment guidelines. Ann Indian Acad Neurol 2011; 14:60-64

7. Kanter DS, Horensky D, Sperling RA, et al. Plasmapheresis in fulminant acute disseminated encephalomyelitis. Neurology 1995; 45:824-827 